\title{
The Classical and Commutative Limits of noncommutative Quantum Mechanics: A Superstar $\star$ Wigner-Moyal Equation
}

\author{
Ardeshir Eftekharzadeh and B. L. Hu \\ Department of Physics, University of Maryland, College Park, Maryland 20742-4111 \\ Received on 5 May, 2005
}

\begin{abstract}
We are interested in the similarities and differences between the quantum-classical (Q-C) and the noncommutative-commutative (NC-Com) correspondences. As one useful platform to address this issue we derive the superstar Wigner-Moyal equation for noncommutative quantum mechanics (NCQM). A superstar $\star$-product combines the usual phase space $*$ star and the noncommutative $\star$ star-product. Having dealt with subtleties of ordering present in this problem we show that the Weyl correspondence of the NC Hamiltonian has the same form as the original Hamiltonian, but with a non-commutativity parameter $\theta$-dependent, momentumdependent shift in the coordinates. Using it to examine the classical and the commutative limits, we find that there exist qualitative differences between these two limits. Specifically, if $\theta \neq 0$ there is no classical limit. Classical limit exists only if $\theta \rightarrow 0$ at least as fast as $\hbar \rightarrow 0$, but this limit does not yield Newtonian mechanics, unless the limit of $\theta / \hbar$ vanishes as $\theta \rightarrow 0$. For another angle towards this issue we formulate the NC version of the continuity equation both from an explicit expansion in orders of $\theta$ and from a Noether's theorem conserved current argument. We also examine the Ehrenfest theorem in the NCQM context.
\end{abstract}

\begin{abstract}
Aim In this program of investigation we ask the question whether there is any structural similarity or conceptual connection between the quantum-classical (Q-C) and the noncommutative-commutative (NC-Com) correspondences. We want to see if our understanding of the quantum-classical correspondence acquired in the last decade can aid us in any way to understand the physical attributes and meanings of a noncommutative space from the vantage point of the ordinary commutative space. We find that the case of quantum to classical transition in the context of noncommutative geometry is quite different from that in the ordinary (commutative) space. Specifically, if $\theta \neq 0$ there is no classical limit. Classical limit exists only if $\theta \rightarrow 0$ at least as fast as $\hbar \rightarrow 0$, but this limit does not yield Newtonian mechanics, unless the limit of $\theta / \hbar$ vanishes as $\theta \rightarrow 0$. We make explicit this relationship by deriving a superstar $\star$ Wigner-Moyal equation for noncommutative quantum mechanics (NCQM) and identifying the difference between the classical and the commutative limits. A superstar $\star$-product combines the usual phase space $*$ star and the noncommutative $\star$-product [1].

In this paper we focus on the nature of the commutative and classical limits of noncommutative quantum physics. We point out some subtleties which arise due to the ordering problem. When these issues are properly addressed we show that the classical correspondent to the NC Hamiltonian is indeed one with a $\theta$-dependent, momentum-dependent shift in the coordinates. For another angle towards this issue we formulate the NC version of the continuity equation both from an explicit expansion in orders of $\theta$ and from a Noether's theorem conserved current argument. We also examine the Ehrenfest theorem in the NCQM context.
\end{abstract}

\section{CRITERIA FOR CLASSICALITY}

We open this discussion by examining the quantum to classical (Q-C) transition issue which is probably more familiar to us than the noncommutative to commutative (NC-Com) tran- sition. We begin by listing the criteria related to the Q-C issue so that we can see the possibilities in how to approach the NCCom issue, if there is some analogy we can draw. In fact the focus of this paper is to ask if any such analogy or parallel exists, both conceptually and structurally. (The following is excerpted from [2])

A quick sampling of discussions in quantum mechanics and statistical mechanics textbooks reveals a variety of seemingly simple and straightforward criteria and conditions for classicality. For example, one can loosely associate:

1) $\hbar \rightarrow 0$

2) WKB approximation, which "gives the semiclassical limit"

3) Ehrenfest Theorem, "quantum expectation follows a classical equation of motion"

3) Wigner function, "behaves like a classical distribution function"

4) high temperature limit: "thermal=classical"

5) Uncertainty Principle: a system "becomes classical" when this is no longer obeyed

6) coherent states: the 'closest' to the classical

7) systems with large quantum number $n \rightarrow \infty$ (correspondence principle)

8 ) systems with large number of components $1 / N \rightarrow 0$.

Each of these conditions contains only some partial truth and when taken on face value can be very misleading. Many of these criteria hold only under special conditions. They can approximately define the classical limit only when taken together in specific ways. To understand the meaning of classicality it is important to examine the exact meaning of these criteria, the conditions of their validity and how they are related to each other.

We can divide the above conditions into four groups, according to the different issues behind these criteria:

a) quantum interference,

b) quantum and thermal fluctuations,

c) choice of special quantum states,

d) meaning of the large $n$ and $N$ limits. 
The first two groups of issues were discussed in [2] using the paradigm of quantum open systems. The first set of issues a) was discussed in the context of quantum cosmology by Habib and Laflamme [3]. They asserted that decoherence is needed for the WKB Wigner function to show a peak in phase space indicating the correlation between the physical variables and their canonical conjugates which defines a classical trajectory. This clarifies the loose connection of WKB, Wigner function and classicality. For issue b), for ordinary systems the time for thermal fluctuations to overtake quantum fluctuations is also related to the time of decoherence. But a decohered system is not necessarily classical. There is a quantum statistical regime in between. This set of issues was addressed by Hu and Zhang [4]. (See also [5, 6].) They derived an uncertainty principle for a quantum open system at finite temperature which interpolates between the (zero temperature) quantum Heisenberg relation and the high temperature result of classical statistical mechanics. This was useful for clarifying the sometimes vague notions of quantum, thermal and classical.

In our current investigation we wish to use what was learned in the last decade in $\mathrm{Q}-\mathrm{C}$ to inquire about a simple yet important issue, namely, under what conditions is the ordinary commutative space a bona fide limit of NC space, or, what is the nature of the NC-Com transition?

Recall for QM:

$$
\left[\hat{x}^{i}, \hat{p}_{j}\right]=i \hbar \delta_{j}^{i}
$$

whereas for NC geometry, two spatial coordinates $x^{i}, x^{j}$ satisfy the relation

$$
\left[\hat{x}^{i}, \hat{x}^{j}\right]=i \theta^{i j}
$$

We will refer to $\theta^{i j}$ or simply $\theta$ as the non-commutativity parameter.

From (2), we can see that the non-commutativity parameter $\theta$ has the dimension of length squared. If the geometry of space-time at a fundamental level is to be noncommutative then one possible candidate for $\sqrt{\theta}$ is the Planck length. This is how quantum gravity is linked with NCG, which also bears a close relation to matrix models, quantum groups, M-theory and string theory [7-13].

Here we will actually work around the simplest criterion 1) $\hbar \rightarrow 0$ limit in QM and ask the parallel question how the $\theta \rightarrow 0$ limit would be different, and how these two limits relate to each other. The place where both Q-C and NC-Com share some nontrivial point of contact, at least formally, is the Weyl correspondence between operators and c-functions, the star product, the Wigner distribution, and the Wigner-Weyl equation. This is the domain of semiclassical or semiquantal physics. We will use this equation and the Wigner-Weyl correspondence to explore the $\mathrm{NC}-\mathrm{Com}$ and the Q-C transition.

\section{QUANTUM-CLASSICAL CORRESPONDENCE}

The Wigner distribution function has found applications in kinetic theory and has been instrumental in studying quantum coherence and quantum to classical transitions. Star product arises from considering the implications of Weyl transformation of quantum canonical operators. (A good introduction to these topics can be found in [14]. A succinct treatment of Moyal Bracket can be found in an Appendix of [15]. Readers familiar with these topics can skip to the next section.)

For simplicity, in what follows, we consider one dimensional motion. The phase space canonical coordinates are denoted by $q$ and $p$ respectively for position and momentum dynamical variables and their corresponding quantum mechanical operators are denoted by $\hat{q}$ and $\hat{p}$.

\section{A. Weyl correspondence}

Weyl [18] proposed that all dynamical variables be written in terms of members of the Lie algebra of transformations given by:

$$
\hat{U}(\lambda, \mu)=e^{i(\lambda \hat{q}+\mu \hat{p}) / \hbar}
$$

Let us define the set of phase-space operators as the set of all operators whose operator properties solely depends on $\hat{q}$ and $\hat{p}$. Throughout this article, a member of this set will be called a phase-space operator. One can show that for such operators we can give the following representation:

$$
\hat{A}(\hat{q}, \hat{p})=\int d \lambda d \mu \alpha(\lambda, \mu) e^{i(\lambda \hat{q}+\mu \hat{p}) / \hbar}
$$

$\alpha(\lambda, \mu)$ can be projected back to $(q, p)$ space by the inverse transformation:

$$
\alpha(\lambda, \mu)=\frac{1}{2 \pi \hbar} \int d q d p A_{W}(q, p) e^{-i(\lambda q+\mu p) / \hbar}
$$

where $A_{W}$ is called the Weyl correspondence of $\hat{A}$. We can combine equations (4) and (5) to obtain:

$$
\hat{A}(\hat{q}, \hat{p})=\frac{1}{(2 \pi \hbar)^{2}} \int d q d p d \lambda d \mu A_{W}(q, p) e^{i\left(\frac{\lambda(\hat{q}-q)+\mu(\hat{p}-p)}{\hbar}\right)}
$$

The relationship defines a mapping from the set of functions of phase-space variables to the set of phase-space operators. By multiplying both sides of (6) by $U^{\dagger}\left(\lambda^{\prime}, \mu^{\prime}\right)$, taking the trace of both sides and making use of the fact that the $U$ transformations can be inverted since

$$
\operatorname{Tr}\left[U(\lambda, \mu) U^{\dagger}\left(\lambda^{\prime}, \mu^{\prime}\right)\right]=2 \pi \hbar \delta\left(\lambda-\lambda^{\prime}\right) \delta\left(\mu-\mu^{\prime}\right) e^{i \frac{\lambda \mu-\lambda^{\prime} \mu^{\prime}}{2 \hbar}}
$$

we can find the inverse of the above mapping [19]. The result is

$$
A_{W}(q, p)=\frac{1}{2 \pi \hbar} \int e^{i(\lambda q+\mu p) / \hbar} \operatorname{Tr}\left[U^{\dagger}(\lambda, \mu) \hat{A}(\hat{q}, \hat{p})\right] d \lambda d \mu
$$

In what follows we show that every phase-space operator denoted by $\hat{A}(\hat{q}, \hat{p})$ can be written as the mapping represented 
by (6). First we note that every such operator is completely determined by its matrix elements taken with respect to any complete basis. Let the set of position eigenstates be such a basis. One can fully represent the operator in question as $\left\langle x_{1}|\hat{A}| x_{2}\right\rangle$. Introduce the following change of variables

$$
x_{1}=X+\Delta, x_{2}=X-\Delta
$$

with inverse

$$
X=\frac{x_{1}+x_{2}}{2}, \Delta=\frac{x_{1}-x_{2}}{2}
$$

one can define

$$
A(X, \Delta) \equiv\left\langle x_{1}|\hat{A}| x_{2}\right\rangle
$$

Every operator can be written in such a way. Next, we use the Fourier theorems to write:

$$
\left\langle x_{1}|\hat{A}| x_{2}\right\rangle=\frac{1}{2 \pi \hbar} \int d p A_{W}(X, p) e^{-i \Delta p / \hbar}
$$

where the use of index $W$ and the connection with the Weyl correspondence will be clarified shortly. Inserting an integral over additional variable, $q$

$$
\begin{aligned}
\left\langle x_{1}|\hat{A}| x_{2}\right\rangle & =\frac{1}{2 \pi \hbar} \int d p d q \delta(X-q) A_{W}(q, p) e^{-i \Delta p / \hbar} \\
& =\frac{1}{(2 \pi \hbar)^{2}} \int d p d q d \lambda e^{i \lambda(X-q) / \hbar} A_{W}(q, p) e^{-i \Delta p / \hbar}
\end{aligned}
$$

we get

$$
\left\langle x_{1}|\hat{A}| x_{2}\right\rangle=\frac{1}{(2 \pi \hbar)^{2}} \int d p d q d \lambda A_{W}(q, p) e^{i \lambda\left(-q+\frac{x_{1}+x_{2}}{2}\right) / \hbar} e^{i\left(x_{1}-x_{2}\right)\left(\frac{\lambda}{2}-p\right) / \hbar}
$$

Now insert another integral over $\delta\left(x_{1}-x_{2}-\mu\right)$ to eliminate $\left(x_{1}-x_{2}\right)$. This latter Dirac delta function can be written as $\left\langle x_{1} \mid x_{2}+\mu\right\rangle$ which is equal to $\left\langle x_{1}\left|e^{i \mu \hat{p} / \hbar}\right| x_{2}\right\rangle$. Once the position eigen kets are inserted,

one can write factors like $\left(e^{i \lambda x_{1} / \hbar}\left\langle x_{1}\right|\right)$ as $\left(\left\langle x_{1}\right| e^{i \lambda \hat{q} / \hbar}\right)$. Combining all of the above we have

$$
\left\langle x_{1}|\hat{A}| x_{2}\right\rangle=\frac{1}{(2 \pi \hbar)^{2}} \int d p d q d \lambda d \mu A_{W}(q, p) e^{-i(\lambda q+\mu p) / \hbar}\left\langle x_{1}\left|e^{\frac{i \lambda \hat{q}}{2 \hbar}} e^{i \mu \hat{p} / \hbar} e^{\frac{i \lambda \hat{q}}{2 \hbar}}\right| x_{2}\right\rangle
$$

Now we know that the $\left|x_{1}\right\rangle$ and $\left|x_{2}\right\rangle$ were arbitrary. If the operator properties of $\hat{A}$ solely depends on $\hat{p}$ and $\hat{q}$, that is, if the collection of all the matrix elements of the type (11) can fully describe the operator $\hat{A}$, then the aforementioned state kets can be omitted from both sides of the equation. Then we can use the Baker-Campbell-Hausdorff lemma to combine operators inside the bra-ket into $e^{(\lambda \hat{q}+\mu \hat{p}) / \hbar}$ and therefore show that any phase-space operator can be written as (6). That is to say, for every phase-space operator, there is a function of the phase-space variables such that the relationship (6) holds. Thus the Weyl correspondence represented by (6) is an onto mapping from the space of functions into the space of phasespace operators. Furthermore one can show that the Weyl correspondence is a one-to-one mapping. To see that let us assume there are two different functions, namely $A_{W}(q, p)$ and $A_{W}^{\prime}(q, p)$ that map to a single operator. That is

$$
\begin{aligned}
& \int d q d p d \lambda d \mu A_{W}(q, p) e^{i\left(\frac{\lambda(\hat{q}-q)+\mu(\hat{p}-p)}{\hbar}\right)} \\
= & \int d q d p d \lambda d \mu A_{W}^{\prime}(q, p) e^{i\left(\frac{\lambda(\hat{q}-q)+\mu(\hat{p}-p)}{\hbar}\right)}
\end{aligned}
$$

Now one can use (7) to reverse both sides and by using the properties of the Fourier transformation can show that $A_{W}(q, p)$ and $A_{W}^{\prime}(q, p)$, are indeed identical. Therefore the Weyl correspondence is a one-to-one and onto mapping from the set of functions over the phase-space variables to the set of phase-space operators as defined at the beginning of this subsection.

\section{B. Wigner Function}

Wigner distribution functions $W(q, p)$ in quantum systems are meant to play the corresponding role of classical distributions in classical kinetic theory. For a classical system in kinetic theory and a positive-definite distribution function $P(q, p)$ of the canonical variables $q, p$ in classical phase space, we have [17]:

$$
\langle A\rangle_{\text {classical }}=\int A(q, p) P(q, p) d q d p
$$


Let us assume that the quantum system is described by the wave function $\psi(x)=\langle x \mid \psi\rangle$. One can define

$$
\begin{aligned}
A(q, p) & =2 \int d x e^{2 i x p / \hbar}\langle q-x|\hat{A}(\hat{q}, \hat{p})| q+x\rangle \\
W(q, p) & =\frac{1}{\pi \hbar} \int d x e^{-2 i x p / \hbar} \psi^{*}(q-x) \psi(q+x)
\end{aligned}
$$

where $W(q, p)$ is called the Wigner function and it can be shown to have the following main property

$$
\langle\hat{A}\rangle_{q u a n t u m}=\int A(q, p) W(q, p) d q d p
$$

Note here that $\hat{A}$ is a phase-space operator. To show that the transformation defined by Eq. (18) is equivalent to the Weyl correspondence we use Eq. (8) to obtain

$$
A_{W}(q, p)=\frac{1}{2 \pi \hbar} \int d \lambda d \mu e^{i(\lambda q+\mu p) / \hbar} \operatorname{Tr}\left[U^{\dagger}(\lambda, \mu) \hat{A}\right] .
$$

The factor involving the trace can be rewritten as,

$$
\begin{aligned}
\operatorname{Tr}\left[U^{\dagger}(\lambda, \mu) \hat{A}\right] & =\int d q^{\prime}\left\langle q^{\prime}\left|e^{-i \lambda \hat{q} / \hbar} e^{-i \mu \hat{p} / \hbar} e^{i \lambda \mu / 2 \hbar} A\right| q^{\prime}\right\rangle \\
& =\int d q^{\prime} e^{-i \lambda q^{\prime} / \hbar} e^{i \lambda \mu / 2 \hbar}\left\langle q^{\prime}\left|e^{-i \mu \hat{p} / \hbar} A\right| q^{\prime}\right\rangle \\
& =\int d q^{\prime} e^{-i \lambda q^{\prime} / \hbar} e^{i \lambda \mu / 2 \hbar}\left\langle q^{\prime}-\mu|A| q^{\prime}\right\rangle
\end{aligned}
$$

which can be substituted back in Eq. (21) to obtain

$$
\begin{aligned}
& A_{W}(q, p) \\
= & \frac{1}{2 \pi \hbar} \int d \lambda d \mu d q^{\prime} e^{i \lambda\left(q-q^{\prime}+\mu / 2\right) / \hbar} e^{i \mu p}\left\langle q^{\prime}-\mu|A| q^{\prime}\right\rangle \\
= & \int d \mu d q^{\prime} \delta\left(q^{\prime}-q-\mu / 2\right) e^{i \mu p}\left\langle q^{\prime}-\mu|A| q^{\prime}\right\rangle \\
= & \int d \mu e^{i \mu p}\langle q-\mu / 2|A| q+\mu / 2\rangle .
\end{aligned}
$$

With a slight change of variable to $x=\mu / 2$ we have

$$
A_{W}(q, p)=2 \int e^{i 2 x p / \hbar}\langle q-x|A| q+x\rangle d x,
$$

which proves the equivalence of the two mappings.

Note that the above transformation has the following property

$$
\int d q d p A(q, p)=2 \pi \hbar \operatorname{Tr}[\hat{A}(\hat{q}, \hat{p})]
$$

and that the Wigner distribution function is actually the Weyl transformation of the density matrix operator $\hat{\rho}=|\psi\rangle\langle\psi|$

$$
\begin{aligned}
\rho_{W}(q, p) & =2 \pi \hbar W(q, p) \\
& =2 \int e^{i 2 \bar{q} p / c}\langle q-\bar{q} \mid \psi\rangle\langle\psi \mid q+\bar{q}\rangle d \bar{q}
\end{aligned}
$$

Unlike the classical case, where a probabilistic interpretation of the distribution function is possible, the Wigner function cannot be interpreted as a probability distribution because in general it is not everywhere positive. Let $|p\rangle$ and $|q\rangle$ be the eigenkets of operators $\hat{p}$ and $\hat{q}$ with eigenvalues $p$ and $q$ respectively. Assuming the system is in the state denoted by $|\psi\rangle$, it can be easily shown that the Wigner function has the following properties:

$$
\int W(q, p) d q=\langle p \mid \psi\rangle\langle\psi \mid p\rangle, \quad \int W(q, p) d p=\langle q \mid \psi\rangle\langle\psi \mid q\rangle
$$

For completeness we note that for a mixed state the Wigner function can be defined as

$$
W(q, p)=\frac{1}{\pi \hbar} \int d x e^{-2 i x p / \hbar} \rho(q-x, q+x)
$$

\section{Phase space $*$-product and Wigner-Moyal equation}

Consider two dynamical variables $A$ and $B$ in a classical system. The statistical average of their product is obtained by weighting it with the distribution function $P(q, p)$ given by

$$
\langle A B\rangle_{\text {classical }}=\int A(q, p) B(q, p) P(q, p) d q d p .
$$

If $A$ and $B$ are quantum mechanical operators, because of their functional dependence on the non-commuting operators $\hat{q}$ and $\hat{p}$ a different rule of multiplication, the star product, is needed. The star product satisfies the following property [1]

$$
\langle\hat{A} \hat{B}\rangle=\int A_{W}(q, p) * B_{W}(q, p) W(q, p) d q d p
$$

Alternatively,

$$
C(q, p)=(\hat{A} \hat{B})_{W}(q, p)=A_{W}(q, p) * B_{W}(q, p)
$$

where the symbol $(.)_{W}$ for products of operators, stands for the Weyl transformation of the enclosed operator inside. How is the star product related to the ordinary algebraic product? To find out we first use the Weyl analysis for the solution

$$
C(q, p)=\frac{1}{2 \pi \hbar} \int e^{i(\lambda q+\mu p) / \hbar} \operatorname{Tr}\left[U^{\dagger}(\lambda, \mu) \hat{A} \hat{B}\right] d \lambda d \mu
$$

We substitute for $\hat{A}$ and $\hat{B}$ from (6) and use the fact that $A_{W}(q, p)=A(q, p), B_{W}(q, p)=B(q, p)$ to write 


$$
C(q, p)=\frac{1}{(2 \pi \hbar)^{4}} \int A\left(q^{\prime}, p^{\prime}\right) e^{i \frac{\lambda^{\prime}\left(q-q^{\prime}\right)+\mu^{\prime}\left(p-p^{\prime}\right)}{\hbar}} e^{\frac{-i}{2 \hbar}\left(\lambda^{\prime} \mu^{\prime \prime}-\lambda^{\prime \prime} \mu^{\prime}\right)} e^{i \frac{\lambda^{\prime \prime}\left(q-q^{\prime \prime}\right)+\mu^{\prime}\left(p-p^{\prime \prime}\right)}{\hbar}} B\left(q^{\prime \prime}, p^{\prime \prime}\right) d q^{\prime} d p^{\prime} d q^{\prime \prime} d p^{\prime \prime} d \lambda^{\prime} d \lambda^{\prime \prime} d \mu^{\prime} d \mu^{\prime \prime}
$$

where we have made use of (7) and

$$
U^{\dagger}(\lambda, \mu) U\left(\lambda^{\prime}, \mu^{\prime}\right)=U^{\dagger}\left(\lambda-\lambda^{\prime}, \mu-\mu^{\prime}\right) e^{i\left(\lambda \mu^{\prime}-\lambda^{\prime} \mu\right) / 2 \hbar} .
$$

The above relationship can be written as:

$$
\begin{aligned}
C(q, p) & =A(q, p) * B(q, p) \\
& =A(q, p) e^{i \frac{\hbar}{2}\left(\frac{\overleftarrow{\partial}}{\partial q} \frac{\vec{\partial}}{\partial p}-\frac{\overleftarrow{\partial}}{\partial p} \frac{\vec{\partial}}{\partial q}\right)} B(q, p)
\end{aligned}
$$

This procedure for combining two functions defines the phase space $*$-product. Another way of writing it is

$$
\begin{aligned}
& A(q, p) * B(q, p)= \\
& \left.e^{\frac{i \hbar}{2}\left(\frac{\partial}{\partial q} \frac{\partial}{\partial p^{\prime}}-\frac{\partial}{\partial p} \frac{\partial}{\partial q^{\prime}}\right)} A(q, p) B\left(q^{\prime}, p^{\prime}\right)\right|_{\left(q^{\prime}, p^{\prime}\right) \rightarrow(q, p)}
\end{aligned}
$$

Using these three entities, namely, Wigner function, Weyl transformation and the star product, we can construct the Wigner-Moyal-Weyl-Groenwood formalism. This formalism has been well developed long before the recent activities in NC geometry and been used widely for the study of semiclassical physics (see, e.g., [14-16]). The state of a quantum system can be represented by a real valued function of the canonical coordinates, the Wigner function. We notice that the star-squared of a Wigner function (for a pure state) is proportional to itself.

$$
W * W=\frac{1}{\hbar} W
$$

The Weyl transformation of the Dirac bracket of two operators can be shown to be equal to their commutator with respect to the star product:

$$
[\hat{A}, \hat{B}]_{W}=[A, B]_{*} \equiv A * B-B * A
$$

It can be shown that using the Weyl transformation of the eigenvalue equation for the density operator, corresponding to an energy eigen state, we obtain:

$$
H * W_{n}=E_{n} W_{n}
$$

The eigenvalue equation is thus formulated as a "star-gen value" equation.

The time evolution of the system's state is governed by the Wigner-Moyal equation. For a Hamiltonian of the form

$$
H(x, p)=\frac{p^{2}}{2 m}+V(x)
$$

The Wigner-Moyal equation is written as

$$
\begin{aligned}
\frac{\partial W}{\partial t} & =-\frac{2}{\hbar} W \sin \frac{\hbar}{2}\left(\frac{\grave{\partial}}{\partial q} \frac{\vec{\partial}}{\partial p}-\overleftarrow{\partial} \frac{\overleftarrow{\partial}}{\partial p}\right) H \\
& =\frac{2}{\hbar}(H * W-W * H)
\end{aligned}
$$

Or, equivalently, from (Eq. 38),

$$
\frac{\hbar}{2} \frac{\partial W}{\partial t}=[H, W]_{*}
$$

So far we have discussed everything in one space dimension. The extension to $N$ dimensional space is straightforward. The commutation relation takes the form:

$$
\left[q^{i}, p^{j}\right]=i \hbar \delta^{i j}
$$

The star product is associative, that is

$$
[(f * g) * h]=[f *(g * h)],
$$

The complex conjugate (c) of the star product of two functions is given by

$$
(f * g)^{c}=g^{c} * f^{c}
$$

Finally the star product of functions under integration exhibits the cyclic property:

$$
\begin{aligned}
& \int\left(f_{1} * f_{2} * \cdots * f_{n}\right)(x) d^{N} q d^{N} p= \\
& \int\left(f_{n} * f_{1} * \cdots * f_{n-1}\right) d^{N} q d^{N} p
\end{aligned}
$$

In particular, for two functions in a $2 \mathrm{~N}$-dimensional phase space ( $\mathrm{N}$ dimensional configuration space), we have

$$
\begin{aligned}
\int(f * g)(x) d^{N} q d^{N} p & =\int(g * f)(x) d^{N} q d^{N} p \\
& =\int(f \cdot g)(x) d^{N} q d^{N} p
\end{aligned}
$$

The last equation states that for two functions of phase space coordinates ( where $\left[\hat{q}^{i}, \hat{p}_{j}\right]=i \hbar \delta_{j}^{i}$ ), the integral of the starproduct over all phase space gives the same result as that obtained by using the ordinary product. (For an introduction to the properties of time-independent Wigner functions see [20]. Our notation in this section follows [17]).

\section{NONCOMMUTATIVE GEOMETRY}

Noncommutative geometry (NCG) has appeared in the literature ever since Heisenberg and Snyder studied it with the hope of resolving the ultraviolet infinity problem [21]. Later on it was applied to the Landau model of electrons in a magnetic field, where considering certain limits (the lowest energy levels) the space of the coordinates becomes a noncommutative space. Recent interest in noncommutative physics, however, stems from the discovery of NCG in the context of string theory and M theory $[8,9]$. NCG has been considered as a 
candidate for Plank scale geometry. Hence, a successful theory of quantum gravity may reveal the necessity or desirability of some form of noncommutative geometry.

There are various approaches to formulate noncommutative geometry. Early attempts using a more mathematical approach were proposed by Alain Connes and John Madore $[22,23]$. In this phase, a differential NC geometry was developed and the concept of distance and differential forms were defined. Later progress focused more around the WignerMoyal formalism, described in the last section. Almost all current work on the subject of fields in noncommutative spaces relies on using star product and its properties. This is the approach pursued here.

\section{A. Noncommutative $\star$-star product}

To introduce non-commutativity, one replaces the normal product between two functions with the $\star$-product defined as

$$
f(x) \star g(x)=f(x) e^{\frac{i \theta^{i j}}{2} \frac{\overleftarrow{\partial}}{\partial x^{i}} \frac{\vec{\partial}}{\partial x^{j}}} g(x)
$$

The $\star$-product inherits all the properties of its phase space counterpart, the $*$-product.

In the previous section we used $q$ as the canonical variable for position. From now on we denote it by $x$. In what follows, we also use the beginning letters of the Latin alphabet, $a, b$ to denote the coordinate indices rather than the middle letters $i, j$. With this we can expand the $\star$-product as

$$
\begin{gathered}
f(x) \star g(x)=f(x) g(x)+\sum_{n=1}\left(\frac{1}{n !}\right)\left(\frac{i}{2}\right)^{n} \times \\
\theta^{a_{1} b_{1}} \ldots \theta^{a_{n} b_{n}} \partial_{a_{1}} \ldots \partial_{a_{k}} f(x) \partial_{b_{1}} \ldots \partial_{b_{k}} g(x)
\end{gathered}
$$

In the interest of brevity, sometimes we put the lower limit of the sum as $n=0$ to replace the first term in the above, i.e., the $n=0$ term is $f(x) g(x)$, without derivatives or $\theta$ dependence.

\section{B. Noncommutative Quantum Mechanics}

Using the non-relativistic limit of noncommutative quantum field theory (NCQFT) (see $[12,25]$ for a review), one can obtain the Schrödinger equation for noncommutative quantum mechanics (NCQM) as follows [24, 26]:

$$
i \hbar \frac{\partial}{\partial t} \psi=-\frac{\nabla^{2}}{2 m} \psi+V \star \psi .
$$

Here we are studying the quantum mechanics of a particle in an external potential. As is well-known, using the form of $\star$-product, one can write the noncommutative part as $V\left(\hat{x}^{i}-\hat{p}_{j} \theta^{i j} /(2 \hbar)\right) \psi(x)$. However one must pay attention to the ordering issues that can arise. To be consistent with the definition of a $\star$-product, the ordering here is such that all momentum operators stand to the right of the rest of the potential and operate directly on the wave function.

The definition of Wigner function does not change in the NC settings. However we expect the time evolution of the Wigner function following the Wigner-Moyal (WM) equation to be different. To obtain the WM equation for NCQM, one can start from (50) in a somewhat cumbersome yet straightforward manner. An easier way is to apply the Weyl correspondence to the von Neumann equation,

$$
i \hbar \frac{d \hat{\rho}}{d t}=\hat{\rho} \hat{H}^{N C}-\hat{H}^{N C} \hat{\rho}
$$

where the NC Hamiltonian is written as:

$$
\hat{H}^{N C}=\frac{\hat{p}^{2}}{2 m}+\sum_{n=0} \frac{1}{n !}\left(\frac{-1}{2 \hbar}\right)^{n} \theta^{a_{1} b_{1}} \cdots \theta^{a_{n} b_{n}} \partial_{a_{1}} \cdots \partial_{a_{n}} V \hat{p}_{b_{1}} \cdots \hat{p}_{b_{n}}
$$

We begin with the equation governing the Wigner function as it is normally defined

$$
-i \hbar \frac{d W}{d t}=W * H_{W}^{N C}-H_{W}^{N C} * W
$$

where $H_{W}^{N C}$ is the Weyl correspondent of the noncommutative Hamiltonian. To find the Weyl transformation we use the usual definition:

$$
H_{W}^{N C}(x, p)=8 \int e^{2 i \mathbf{y} \cdot \mathbf{p} / \hbar}\langle x-y|\hat{H}| x+y\rangle d^{3} y
$$

For convenience let us define

$$
\begin{array}{cc}
A(x)^{b_{1} \ldots b_{n}} & = \\
& \left(-\frac{1}{2 \hbar}\right)^{n} \quad \theta^{a_{1} b_{1}} \cdots \theta^{a_{n} b_{n}} \partial_{a_{1}} \cdots \partial_{a_{n}} V(x)
\end{array}
$$

Then we have

$$
\begin{aligned}
H_{W}^{N C}(x, p) & =\frac{p^{2}}{2 m}+8 \sum_{n=0} \frac{1}{n !} \\
& \int e^{2 i \mathbf{y} \cdot\left(\mathbf{p}-\mathbf{p}^{\prime}\right) / \hbar} A(x-y)^{b_{1} \ldots b_{n}} p_{b_{1}}^{\prime} \cdots p_{b_{n}}^{\prime} d^{3} p^{\prime} d^{3} y
\end{aligned}
$$


which can be shown to be equivalent to

$$
H_{W}^{N C}(x, p)=\frac{p^{2}}{2 m}+8 \sum_{n=0} \frac{1}{n !} \prod_{k=1}^{n}\left(p_{b_{k}}-\frac{\hbar}{i} \partial_{b_{k}}\right) A(x)^{b_{1} \ldots b_{n}}
$$

But since $\theta^{a b} \partial_{a} \partial_{b} V$ vanishes, after expanding the product no derivative survives and we get

$$
H_{W}^{N C}(x, p)=H_{W}\left(x^{a}-\frac{\theta^{a b}}{2 \hbar} p_{b}, p\right)
$$

That is, the Weyl transformation of $H(\hat{x}, \hat{p})$ has the same functional form in terms of $x$ and $p$ as the commutative Hamiltonian but with position $x^{a}$ shifted by an amount equal to $-\frac{\theta^{a b}}{2 \hbar} p_{b}$, where $p_{b}$ is the phase space momentum.

\section{A Superstar $\star$ Wigner-Moyal equation}

With the change of coordinates,

$$
\begin{aligned}
& x^{\prime a}=x^{a}-\frac{\theta^{a b}}{2 \hbar} p_{b} \\
& p^{\prime a}=p^{a}
\end{aligned}
$$

we can rewrite the above equation in a more suggestive form as:

$$
\begin{aligned}
-i \hbar \frac{\partial \tilde{W}}{\partial t}= & \quad \tilde{W}\left(x^{\prime}, p^{\prime}\right) \star H_{W}\left(x^{\prime}, p^{\prime}\right) \\
& -H_{W}\left(x^{\prime}, p^{\prime}\right) \star \tilde{W}\left(x^{\prime}, p^{\prime}\right)
\end{aligned}
$$

with

$$
\begin{aligned}
\tilde{W}\left(x^{\prime}, p^{\prime}\right) & =W\left(x^{\prime a}+\frac{\theta^{a b}}{2 \hbar} p_{b}^{\prime}, p^{\prime a}\right) \\
\star & \equiv e^{\frac{i \hbar}{2}\left(\overleftarrow{\partial}_{\mathbf{x}^{\prime}} \cdot \vec{\partial}_{\mathbf{p}^{\prime}}-\overleftarrow{\partial}_{\mathbf{p}^{\prime}} \cdot \vec{\partial}_{\mathbf{x}^{\prime}}\right)+\frac{i \theta^{a b}}{2} \frac{\overleftarrow{\partial}}{\partial x^{\prime a}} \frac{\vec{\partial}}{\partial x^{\prime b}}}
\end{aligned}
$$

where $x, x^{\prime}, p$ and $p^{\prime}$ are phase space variables, not operators. This is the main mathematical result of this paper.

In related works, Jing et al [28] had derived an explicit form for the Wigner functions in NCQM and showed that it satisfies a generalized *-genvalue equation. (We thank Dr. J. Prata for bringing to our attention this reference.) Dayi and Kelleyane [29] derived the Wigner functions for the Landau problem when the plane is noncommutative. They introduced a generalized $*$-genvalue equation for this problem and found solutions for it.

Now we use this equation to examine the classical and commutative limits. In the limit of small $\hbar$ the equation(61) becomes

$$
\begin{aligned}
\frac{\partial \tilde{W}}{\partial t}= & \frac{1}{2} \quad\left(\partial_{x^{\prime i}} H \star \partial_{p^{\prime i}} \tilde{W}-\partial_{p^{\prime i}} H \star \partial_{x^{\prime i}} \tilde{W}\right)- \\
& \frac{1}{2}\left(\partial_{x^{\prime i}} \tilde{W} \star \partial_{p^{\prime i}} H-\partial_{p^{\prime i}} \tilde{W} \star \partial_{x^{\prime i}} H\right)+ \\
& \frac{1}{i \hbar} \quad(H \star \tilde{W}-\tilde{W} \star H)
\end{aligned}
$$

From this we conclude that if $\theta$ is kept $\neq 0$ the classical limit $(\hbar \rightarrow 0)$ does not exist. In order for the classical limit of NCQM to exist, $\theta$ must be of order $\hbar$ or higher, or, if $\theta \rightarrow 0$ at least as fast as $\hbar \rightarrow 0$. Note, however, that this limit does not yield Newtonian mechanics, unless the limit of $\theta / \hbar$ vanishes as $\theta \rightarrow 0$.

Comparing with earlier claims on this issue, a different conclusion was reached by Acatrinei [31] who proposed a phasespace path integral formulation of NCQM which "suggests that a classical limit always exists" (communication from the cited author).

Also relevant to our finding here are earlier results from perturbative noncommutative field theories. For scalar theories, non-planar diagrams lead to infra-red divergences [32, 33] which renders the theory singular in the $\theta \rightarrow 0$ limit. This situation also arise in gauge theories (e.g., [34]). In studies of perturbative NC Yang-Mills theory, e.g., Armoni [35] pointed out that even at the planar limit the $\theta \rightarrow 0$ limit of the $\mathrm{U}(\mathrm{N})$ theory does not converge to the ordinary $S U(N) \times U(1)$ commutative theory. This is due to the renormalization procedure being incommensurate with noncommutativity. This is also related to the IR/UV issue in string theory. (There is a huge literature on NC field theory. For reviews, see, e.g., [12, 25])

\section{CONTINUITY EQUATION AND EHRENFEST THEOREM}

To further explore the classical and commutative $(\hbar \approx 0$, $\theta \approx 0$ ) limits, it is instructive to find out the noncommutative version of the continuity equation and that of the Ehrenfest theorem in such a context. Noncommutative classical mechanics and expectation values of quantum mechanical quantities have been studied in [30] (see earlier references therein).

\section{A. Continuity Equation}

From (50) we have

$$
\begin{gathered}
\frac{\partial\left(\psi^{*} \psi\right)}{\partial t}+\nabla \cdot \frac{\hbar}{2 i m}\left(\psi \nabla \psi^{*}-\psi^{*} \nabla \psi\right) \\
-\frac{1}{i \hbar}\left(\psi^{*}(V(x) \star \psi)-\left(\psi^{*} \star V(x)\right) \psi\right)=0
\end{gathered}
$$

To first order in $\theta$, the approximation yields

$$
\begin{aligned}
& \frac{\partial}{\partial t}\left(\psi^{*} \psi\right)+\nabla . \\
& \quad\left(\frac{\hbar}{2 i m}\left(\psi \nabla \psi^{*}-\psi^{*} \nabla \psi\right)+\frac{1}{2 \hbar} V(x)\left(\vec{\theta} \times \nabla\left(\psi^{*} \psi\right)\right)\right) \\
& =0,
\end{aligned}
$$

where we have defined $\left(\hat{x}^{k}\right.$ being the $k^{\text {th }}$ unit vector)

$$
\begin{aligned}
\vec{\theta} & =\theta_{k} \hat{x^{k}} \\
\theta_{k} & =\varepsilon_{i j k} \theta^{i j}
\end{aligned}
$$


To this order our semi-commutative continuity equation does suggest the following quantity as the conserved probability current.

$$
\begin{aligned}
\vec{J}_{(1)}= & \frac{\hbar}{2 i m}\left(\psi \nabla \psi^{*}-\psi^{*} \nabla \psi\right) \\
+ & \frac{1}{2 \hbar} V(x)\left(\vec{\theta} \times \nabla\left(\psi^{*} \psi\right)\right)
\end{aligned}
$$

The existence of a continuity equation for all orders of $\theta$ can be inferred as follows. The $\theta$-dependent term is proportional to

$$
\psi^{*}(V(x) \star \psi)-\left(\psi^{*} \star V(x)\right) \psi
$$

It can be shown that the difference between $A \star B$ and $A B$ is a total divergence. Using this, we can write:

$$
\begin{aligned}
& \psi^{*}(V(x) \star \psi)-\left(\psi^{*} \star V(x)\right) \psi \\
= & \psi^{*} \star V(x) \star \psi+\partial_{i} Q^{i}-\psi^{*} \star V(x) \star \psi-\partial_{i} S^{i} \\
= & \partial_{i}\left(Q^{i}-S^{i}\right)
\end{aligned}
$$

which shows that the $\theta$ dependent term is also a total divergence. In fact one can explicitly compute the conserved current to all orders. To calculate the $n^{\text {th }}$ order term, we consider the last two terms of (65), where

$$
\begin{aligned}
& {\left[\left(\psi^{*}(V(x) \star \psi)-\left(\psi^{*} \star V(x)\right) \psi\right)\right]_{n^{t h} \text { order }}=\left(\frac{1}{n !}\right)\left(\frac{i}{2}\right)^{n} \theta^{a_{1} b_{1}} \ldots \theta^{a_{n} b_{n}} \partial_{a_{1}} } \\
& {\left[\left(\psi^{*} \partial_{a_{2}} \cdots \partial_{a_{n}} V+(-1)^{n-1} V \partial_{a_{2}} \cdots \partial_{a_{n}} \psi^{*}+\sum_{k=2}^{n-1} \partial_{a_{2}} \cdots \partial_{a_{k}} \psi^{*} \partial_{a_{k+1}} \cdots \partial_{a_{n}} V\right) \partial_{b_{1}} \cdots \partial_{b_{n}} \psi-(-1)^{n} \text { c.c. }\right] } \\
+ & V\left(\partial_{a_{1}} \cdots \partial_{a_{n}} \psi^{*} \partial_{b_{1}} \cdots \partial_{b_{n}} \psi-(-1)^{n} \partial_{a_{1}} \cdots \partial_{a_{n}} \psi \partial_{b_{1}} \cdots \partial_{b_{n}} \psi^{*}\right) .
\end{aligned}
$$

Now the two terms in the last line cancel each other, since we can swap all $a$ indices with $b$ indices and then bring back the $\theta$ matrices to their original order by multiplying it by $(-1)^{n}$.
The $n^{\text {th }}$ order $(n \geq 2)$ result in terms of $\theta$ for the conserved current is then given by

$$
\begin{aligned}
J_{(n)}^{a_{1}}=\left(\frac{1}{i \hbar}\right) & \left(\frac{1}{n !}\right)\left(\frac{i}{2}\right)^{n} \theta^{a_{1} b_{1}} \ldots \theta^{a_{n} b_{n}} \times \\
& {\left[\left(\psi^{*} \partial_{a_{2}} \cdots \partial_{a_{n}} V+(-1)^{n-1} V \partial_{a_{2}} \cdots \partial_{a_{n}} \psi^{*}+\sum_{k=2}^{n-1} \partial_{a_{2}} \ldots \partial_{a_{k}} \psi^{*} \partial_{a_{k+1}} \ldots \partial_{a_{n}} V\right) \partial_{b_{1}} \ldots \partial_{b_{n}} \psi-(-1)^{n} \text { c.c. }\right] }
\end{aligned}
$$

In the classical limit all terms must diverge unless $\theta$ is of order $\hbar$ or higher. One plausible argument is to assume that $\theta \sim \ell_{p}^{2}$, where $\ell_{p}$ is the Planck length $\left(\ell_{p}=\sqrt{\hbar G / c^{3}}\right)$. In that case no term will diverge, all terms of higher power in $\theta$ will vanish and the first order term will be non-zero and proportional to $G / c^{3}$.

\section{B. Noether's theorem and Conserved Current}

Instead of performing an explicit expansion in order of $\theta$, one can use a symmetry argument to derive a conserved current in NCQM. Conservation is normally linked to continuous symmetries of the Lagrangian through Noether's theorem. One may try to trace back both the commutative and noncommutative continuity equations to the symmetries of a Lagrangian that produces the equations of motion, namely the Schrödinger equation and its complex conjugate. The Lagrangian for QM can be written as:

$$
L=-\frac{\hbar^{2}}{2 m} \nabla \psi^{*} \cdot \nabla \psi+\frac{i \hbar}{2}\left(\psi^{*} \dot{\psi}-\dot{\psi}^{*} \psi\right)+V \psi \psi^{*} .
$$

This Lagrangian remains invariant under the following transformations

$$
\begin{aligned}
\delta \psi & =i \varepsilon \psi \\
\delta \psi^{*} & =-i \varepsilon \psi^{*}
\end{aligned}
$$

The usual continuity equation in commutative QM is a consequence of Noether's theorem.

It can be shown (for example through expanding the star product and deriving the equations of motion, order by order) 
that the following Lagrangian results in the noncommutative version of the Schrödinger equation and its complex conjugate (i.e. eq. (50))

$$
\begin{aligned}
L=-\frac{\hbar^{2}}{2 m} \nabla \psi^{*} \cdot \nabla \psi & +\frac{i \hbar}{2}\left(\psi^{*} \dot{\psi}-\dot{\psi}^{*} \psi\right) \\
& +\psi^{*} \star V \star \psi
\end{aligned}
$$

One can see that this noncommutative Lagrangian exhibits the same symmetry as the commutative Lagrangian, and thus it admits a conserved current to all orders of $\theta$.

\section{The Ehrenfest theorem}

Another way to explore the relation between quantum and classical mechanics is the Ehrenfest theorem. What is its form in NCQM when $\theta \neq 0$ ? We begin with the time evolution of the expectation value of $\hat{\mathbf{x}}$ to lowest non-vanishing order of $\theta$.
This is given by

$$
\frac{d\left\langle\hat{x}^{i}\right\rangle}{d t}=\frac{\left\langle\hat{p}^{i}\right\rangle}{m}+\frac{\theta^{i j}}{\hbar}\left\langle\partial_{j} V\right\rangle
$$

One can calculate this equation to all orders of $\theta$ Intuitively one can see that the form of Ehrenfest's theorem for position follows simply from the assumption that the noncommutative Hamiltonian can be thought of as a commutative one in which the potential function is evaluated at a shifted position, in a manner that was discussed above (with the appropriate ordering). A direct calculation from first principles confirms this view and we have:

$$
\frac{d\left\langle x^{k}\right\rangle}{d t}=\left\langle\frac{\partial}{\hat{p}_{k}}\left(\frac{\hat{p}^{2}}{2 m}+V\left(\hat{x}^{a}-\frac{\theta^{a b} \hat{p}_{b}}{2 \hbar}\right)\right)\right\rangle
$$

In fact, generally speaking, one can write the equation of motion for the expectation value of any function of canonical operators as:

$$
\frac{d}{d t}\left\langle f\left(\hat{x}^{i}, \hat{p}_{j}\right)\right\rangle=\left\langle\frac{\partial f}{\partial \hat{x}^{k}} \frac{\partial H\left(\hat{x}^{a}-\frac{\theta^{a b}}{2 \hbar} \hat{p}_{b}\right)}{\partial \hat{p}_{k}}-\frac{\partial H\left(\hat{x}^{a}-\frac{\theta^{a b}}{2 \hbar} \hat{p}_{b}\right)}{\partial \hat{x}^{k}} \frac{\partial f}{\partial \hat{p}_{k}}\right\rangle
$$

From this perspective one says that the system will behave classically if

$$
\begin{aligned}
&\left\langle\frac{\partial V(\hat{x})}{\partial \hat{x}^{a}}\right\rangle \approx \frac{\partial V(\langle\hat{x}\rangle)}{\partial\left\langle\hat{x}^{a}\right\rangle} \\
&\left\langle\partial_{a} \partial_{a_{1}} \cdots \partial_{a_{n}} V \frac{\theta^{a_{1} b_{1}} \hat{p}_{b_{1}}}{2 \hbar} \cdots \frac{\left.\theta^{a_{n} b_{n}} \hat{p}_{b_{n}}\right\rangle}{2 \hbar}\right\rangle \frac{\partial^{n} V(\langle\hat{x}\rangle)}{\partial\left\langle\hat{x}^{a}\right\rangle \partial\left\langle\hat{x}^{a_{1}}\right\rangle \cdots \partial\left\langle\hat{x}^{\left.a_{n}\right\rangle}\right.}\left(\frac{\theta^{a_{1} b_{1}}\left\langle\hat{p}_{b_{1}}\right\rangle}{2 \hbar} \cdots \frac{\theta^{a_{n} b_{n}}\left\langle\hat{p}_{b_{n}}\right\rangle}{2 \hbar}\right)
\end{aligned}
$$

These approximations improve if we consider a typical wave packet with a spread of $\Delta x$ in position space and a spread of $\Delta p$ in momentum space, satisfying the following conditions as $\hbar \rightarrow 0$ :

$$
\begin{aligned}
& \Delta x \ll\left|V\left(\frac{\partial V}{\partial x}\right)^{-1}\right| \\
& \Delta p \ll\left|V\left(\frac{\partial V}{\partial p}\right)^{-1}\right|
\end{aligned}
$$

Furthermore the uncertainty principle implies that

$$
\frac{\hbar}{2}<\Delta x \Delta p \ll V^{2}\left|\left(\frac{\partial V}{\partial x} \frac{\partial V}{\partial p}\right)^{-1}\right|
$$

Going back to (77) we again observe that the classical limit does not exist unless $\theta$ goes to zero at least as fast as $\hbar \rightarrow$ 0 . Another important observation is that the classical limit is NOT Newtonian mechanics, unless the ratio $\theta / \hbar$ goes to zero as $\theta \rightarrow 0$. In fact, assuming that $\theta \sim \ell_{p}^{2}$, the limit of $\hbar \rightarrow 0$ gives

$$
\frac{d\left\langle\hat{x}^{i}\right\rangle}{d t} \sim \frac{\left\langle\hat{p}^{i}\right\rangle}{m}+\frac{G}{c^{3}} \varepsilon^{i j}\left\langle\partial_{j} V\right\rangle .
$$

Summary In this note we have given a derivation of the Wigner-Moyal equation under a superstar $\star$ product, which combines the phase space $*$ and the noncommutative $\star$ - star products. We find that the NC-Com $(\theta \rightarrow 0)$ limit is qualitatively very different from the classical $(\hbar \rightarrow 0)$ limit. If $\theta \neq 0$ there is no classical limit. Classical limit exists only if $\theta \rightarrow 0$ at least as fast as $\hbar \rightarrow 0$, but this limit does not yield Newtonian mechanics, except when $\theta / \hbar$ vanishes in the limit of $\theta \rightarrow 0$.

A longer paper addressing additional aspects of this issue is in preparation [36].

Acknowledgement This work would not have been possible without the active participation of Dr. Albert Roura. BLH takes pleasure to thank him for providing important insights, and AE for his critical comments and technical help. BLH and AR would also like to thank Dr. Pei-Ming Ho for a useful correspondence on some technical point in NCQM. This work is supported in part by NSF grant PHY03-00710. 
[1] E. P. Wigner, Phys. Rev. 40, 749 (1932). H. J. Groenewold, Physica 12, 405 (1946). J. E. Moyal, Proc. Cambridge Phil. Soc. 45, 99 (1949).

[2] B. L. Hu, "Quantum and Thermal Fluctuations, Uncertainty Principle, Decoherence and Classicality" Invited Talk at the Third International Workshop on Quantum Nonintegrability, Drexel University, Philadelphia, May, 1992. Published in Quantum Dynamics of Chaotic Systems, edited by J. M. Yuan, D. H. Feng and G. M. Zaslavsky (Gordon and Breach, Philadelphia, 1993) [arXiv:gr-qc/9302029]

[3] S. Habib and R. Laflamme, Phys. Rev. 42, 4056 (1990).

[4] B. L. Hu and Y. Zhang, Mod. Phys. Lett. A8, 3575 (1993); Int. J. Mod. Phys. 10, 4537 (1995).

[5] A. Anderson and J. J. Halliwell, Phys. Rev. D 48 2753 (1993) [arXiv:gr-qC/9304025] . C. Anastopoulos and J. J. Halliwell, Phys. Rev. D 51, 6870 (1995) [arXiv:gr-qc/9407039] . C. Anastopoulos, Phys. Rev. E 53, 4711 (1996) [arXiv:quant-ph/9506031]

[6] W. H. Zurek, J. P. Paz, and S. Habib, Phys. Rev. Lett. 70, 1187 (1993)

[7] A. Connes, M. R. Douglas, and A. Schwarz, "Noncommutative geometry and matrix theory: Compactification on tori,", JHEP 9802, 003 (1998) [arXiv:hep-th/9711162]

[8] E. Witten, "Noncommutative Geometry And String Field Theory," Nucl. Phys. B 268, 253 (1986).

[9] N. Seiberg and E. Witten, "String Theory and Noncommutative Geometry", JHEP 9909 (1999) 032 [arXiv:hep-th/ 9908142].

[10] A. Konechny and A. Schwarz, "Introduction to M(atrix) theory and noncommutative geometry. Parts I and II," Phys. Rept. 360, 353 (2002) [arXiv:hep-th/0012145] , [arXiv:hep-th/0107251]

[11] M. R. Douglas, "Two Lectures on D-Geometry and Noncommutative Geometry", in: Nonperturbative Aspects of String Theory and Supersymmetric Gauge Theories, eds. M. J. Duff, E. Sezgin, C. N. Pope, B. R. Greene, J. Louis, K. S. Narain, S. Randjbar-Daemi, and G. Thompson (World Scientific, Singapore, 1999), p. 131 [arXiv:hep-th/9901146]

[12] Michael R. Douglas and Nikita A. Nekrasov, "Noncommutative Field Theory" [arXiv:hep-th/0106048]

[13] S. Majid, "Hopf Algebras for Physics at the Planck Scale", Class. Quant. Grav. 5, 1587 (1988). S. Majid, Foundations of Quantum Group Theory, (Cambridge University Press, Cambridge 1995).

[14] R. Liboff, Kinetic Theory, (John Wiley, New York, 1998).

[15] L. E. Reichl, The Transition to Chaos: Conservative Classical Systems and Quantum Manifestations (Springer-Verlag, 2004).

[16] Martin C. Gutzwiller, Chaos in Classical and Quantum Me- chanics (Interdisciplinary Applied Mathematics) (SpringerVerlag, Berllin, 1991).

[17] M. Hillery, R. F. O'Connell, M. O. Scully, and E. P. Wigner,"Distribution Functions In Physics: Fundamentals," Phys. Rept. 106, 121 (1984).

[18] H. Weyl, The Theory of Groups and Quantum Mechanics, Dover, New York (1950).

[19] J. A. Harvey,"Komaba lectures on noncommutative solitons and D-branes," [arXiv:hep-th/0102076]

[20] T. Curtright, D. Fairlie, and C. K. Zachos, Phys. Rev. D 58, 025002 (1998) [arXiv:hep-th/9711183]

[21] H. Snyder, Phys. Rev. 71, 38 (1947).

[22] A. Connes, Noncommutative Geometry (Academic, New York, 1994).

[23] J. Madore, An introduction to noncommutative differential geometry and its physical applications No. 257 in London Mathematical Society Lecture Note Series. 2nd edn. (Cambridge University, 1999).

[24] M. Chaichian, M. M. Sheikh-Jabbari, and A. Tureanu. Phys. Rev. Lett. 862716.

[25] R. J. Szabo, Phys. Rep. 378, 207 (2003).

[26] P. M. Ho and H. C. Kao, "Noncommutative quantum mechanics from noncommutative quantum field theory," http://link.aps.org/abstract/PRL/v88/e151602 Phys. Rev. Lett. 88, 151602 (2002) http://xxx.lanl.gov/abs/hep-th/0110191[arXiv:hepth/0110191].

[27] G. D. Barbosa, "On the meaning of the string inspired noncommutativity and its implications," http://jhep.sissa.it/archive/papers/jhep052003024/ jhep052003024.pdf, JHEP 0305, 024 (2003).

[28] S. C. Jing, F. Zuo and T. H. Heng, JHEP 0410, 049 (2004).

[29] O. F. Dayi and L. T. Kelleyane, Mod. Phys. Lett. A 17, 1937 (2002) [arXiv:hep-th/0202062].

[30] A.E.F. Djemai, "On Noncommutative Classical Mechanics", [arXiv:hep-th/0309034]

[31] C. Acatrinei, JHEP 0109, 007 (2001)

[32] D. Bigatti and L. Susskind, [arXiv:hep-th/9908056]

[33] S. Minwalla, M. Van Raamsdonk, and N. Seiberg, "Noncommutative Perturbative Dynamics", [arXiv: hep-th/9912072] M. V. Raamsdonk and N. Seiberg, JHEP 0003 (2000) 035.

[34] e.g., M. Hayakawa, Phys. Lett. B 478, 394 (2000); [arXiv: hep-th/9912167] . A. Matusis, L. Susskind, and N. Toumbas, [arXiv:hep-th/0002075]

[35] A. Armoni, Nucl. Phys. B 593, 229 (2001).

[36] Ardeshir Eftekharzadeh, B. L. Hu, and Albert Roura, "Noncommutative Geometry and Quantum-Classical Correpondence" (in preparation). 